\title{
Interactions of penny-shaped cracks in three-dimensional solids
}

\author{
Shige Zhan · Tzuchiang Wang
}

Received: 3 November 2005 / Accepted: 20 February 2006 / Published online: 15 June 2006

(C) Springer-Verlag 2006

\begin{abstract}
The interaction of arbitrarily distributed penny-shaped cracks in three-dimensional solids is analyzed in this paper. Using oblate spheroidal coordinates and displacement functions, an analytic method is developed in which the opening and the sliding displacements on each crack surface are taken as the basic unknown functions. The basic unknown functions can be expanded in series of Legendre polynomials with unknown coefficients. Based on superposition technique, a set of governing equations for the unknown coefficients are formulated from the traction free conditions on each crack surface. The boundary collocation procedure and the average method for crack-surface tractions are used for solving the governing equations. The solution can be obtained for quite closely located cracks. Numerical examples are given for several crack problems. By comparing the present results with other existing results, one can conclude that the present method provides a direct and efficient approach to deal with three-dimensional solids containing multiple cracks.
\end{abstract}

Keywords Three-dimensional problem .

Penny-shaped cracks $\cdot$ Interaction

The English text was polished by Keren Wang.

\footnotetext{
S. Zhan

Department of Mathematical and Physical Sciences, NSFC, Beijing 100085, China

e-mail: zhansg@nsfc.gov.cn

T. Wang $(\varangle)$

LNM, Institute of Mechanics, Chinese Academy of Sciences,

Beijing 100080, China

e-mail: tcwang@imech.ac.cn
}

\section{Introduction}

Generally, brittle materials contain large numbers of microcracks. Due to the presence of these microcracks, the materials become weaker and less stiff. This problem is of considerable interest for researchers in the fields of solid mechanics, geophysics and materials. Comprehensive reviews on this subject are given by Kachanov [1,2], Nemat-Nasser and Hori [3] and Krajcinovic [4].

In micromechanical fields, due to the complexity of the analysis of the multiple microcracks, various approximate methods have been proposed, such as the dilute or non-interacting solution, the self-consistent method (see e.g. Budiansky and O'Connell [5]), the generalized self-consistent model (see e.g. Christensen and Lo [6]), and the differential scheme (see e.g. Hashin [7]) and Mori-Tanaka method (see e.g. Mori and Tanaka [8]). In these methods, microcrack interactions are entirely neglected or indirectly accounted, which are only valid for low or moderate crack density, since locations of microcracks, and, therefore, the damage and fracture process of brittle materials are not well considered. As crack density increases and microcrack spacings are smaller, the mutual positions of cracks become important and strong interactions between microcracks should be considered. However, it is much more complicated to consider the interaction of cracks than to analyze a problem of single crack. Serious mathematical difficulties arise when we try to consider the interaction of cracks in three-dimensional solids. Due to those reasons, a relatively small number of publications concerning this kind of problems can be found from the literature.

For two coplanar penny-shaped cracks under normal loading, Collins [9] has reduced the problem to an 
infinite set of simultaneous linear Fredholm integral equations, which he solved approximately by iteration for the case when the distances between the cracks are large compared with their radii. A different approach was used by Andreikiv and Panasyuk [10] who reduced the problem to a finite set of integral equations, with the number of equations equal to the number of cracks, but they also managed to consider only the case of cracks far apart. An alternating method was used by O'Donoghue et al. [11] for two or three coplanar elliptical cracks under mode I loading, the results were restricted to spacings larger than one-fourth of the minor axis of the ellipse. The interactions of two parallel elliptical cracks in an infinite solid subjected to uniform tension normal to the crack surfaces were studied by Isida et al. [12], and the smallest spacing between cracks for which numerical results were given was one-fourth of the crack diameter. Based on the superposition principle of elasticity theory and Eshelby's equivalent inclusion method, Xiao et al. [13-16] studied several problems of two interacting penny-shaped or elliptical cracks in three-dimensional solid and analyzed the interaction between a spherical inhomogeneity and two coplanar penny-shaped cracks. In addition, Xiao et al. [17] also investigated the problem of a hoop-like craze formed at the equator of a spherical inhomogeneity.

Strong interactions were studied by Fabrikant [18] and Kachanov [19]. Based on Fabrikant's earlier results for a single circular crack, a new form of integral equations for the problem of coplanar cracks was proposed by Fabrikant [18]; with the advantage that the equations are non-singular and the iteration procedure is rapidly convergent even for interactions of very closely located cracks. Three-dimensional elastic interactions of arbitrarily located and oriented penny-shaped cracks were considered by Kachanov and Laures [20], and solutions for the stress intensity factors (SIFs) were obtained for several crack geometries. Kachanov's method is simple, and can be used for high microcrack concentrations. But in his interaction scheme, the unknown crack-surface tractions are approximated only by their averages.

In this paper, based on the basic equations of elastostatics and the oblate spheroidal coordinates, the displacement functions are expressed in Legendre polynomial series with a set of unknown coefficients for each crack. By superposition technique, using the traction free conditions on each crack surface, a set of governing equations are formulated. The boundary collocation procedure and the average method for crack-surface tractions are used for solving the governing equations. The SIFs are given for several crack geometries and compared with other available results.

\section{Basic formulae and calculation method}

\subsection{Basic formulae}

\subsubsection{A single crack}

The mathematical formulation of three-dimensional elastostatic problems involves an appropriate selection of harmonic functions based upon the solution of the Navier displacement equations of equilibrium. In the absence of body forces, Navier's equations become

$$
\begin{aligned}
& \mu \nabla^{2} u_{i}+(\lambda+\mu) \vartheta_{, i}=0 \quad(i=1,2,3), \\
& \vartheta=u_{i, i},
\end{aligned}
$$

where $u_{i}$ is the component of the displacement vector, $\mu$ the shear modulus, $v$ the Poisson ratio, $\lambda$ the Lamé coefficient, and $\lambda=2 v \mu /(1-2 v)$.

Using the solution of Papkovich and Neuber (Sokolnikoff [21])

$2 \mu u_{i}=\kappa \varphi_{i}-x_{j} \varphi_{j, i}-\varphi_{0, i}$,

where $\varphi_{k}(k=0,1,2,3)$ are harmonic functions, $\kappa=$ $3-4 v$.

Suppose that $F=\varphi_{0}+x_{j} \varphi_{j}$, we rewrite Eq. (2) in the form

$2 \mu u_{i}=4(1-v) \varphi_{i}-\frac{\partial F}{\partial x_{i}}$.

2.1.1.1 Normal loading Based on the work of Kassir and Sih [22], by setting $\varphi_{1}=\varphi_{2}=0, \varphi_{3}=\psi, \varphi_{0}=\varphi$, $F=\varphi+z \psi$ and $\partial \varphi / \partial z=(1-2 v) \psi$ in Eq. (3), the expressions for the corresponding displacements and stresses are

$$
\begin{aligned}
u_{x} & =-\frac{1}{2 \mu}\left(\frac{\partial \varphi}{\partial x}+z \frac{\partial \psi}{\partial x}\right), \\
u_{y} & =-\frac{1}{2 \mu}\left(\frac{\partial \varphi}{\partial y}+z \frac{\partial \psi}{\partial y}\right), \\
u_{z} & =\frac{1}{2 \mu}\left[2(1-v) \psi-z \frac{\partial \psi}{\partial z}\right], \\
\sigma_{x x} & =-\frac{1}{1-2 v}\left(\frac{\partial^{2} \varphi}{\partial x^{2}}+2 v \frac{\partial^{2} \varphi}{\partial y^{2}}+z(1-2 v) \frac{\partial^{2} \psi}{\partial x^{2}}\right), \\
\sigma_{y y} & =-\frac{1}{1-2 v}\left(2 v \frac{\partial^{2} \varphi}{\partial x^{2}}+\frac{\partial^{2} \varphi}{\partial y^{2}}+z(1-2 v) \frac{\partial^{2} \psi}{\partial y^{2}}\right), \\
\sigma_{z z} & =\frac{\partial \psi}{\partial z}-z \frac{\partial^{2} \psi}{\partial z^{2}},
\end{aligned}
$$


$\sigma_{x y}=-\frac{\partial^{2} \varphi}{\partial x \partial y}-z \frac{\partial^{2} \psi}{\partial x \partial y}$

$\sigma_{y z}=-z \frac{\partial^{2} \psi}{\partial y \partial z}$

$\sigma_{x z}=-z \frac{\partial^{2} \psi}{\partial x \partial z}$

The oblate spheroidal coordinates (see Sect. 5) are used to solve three-dimensional elastic interactions of pennyshaped cracks. The harmonic function $\psi$ must satisfy Laplace's equation (44). Its separable solution can be written as

$\psi(\xi, \eta, \theta)=Q_{n}^{m}(\mathrm{i} \xi) P_{n}^{m}(\eta)\left(b_{m n} \cos m \theta+c_{m n} \sin m \theta\right)$,

where $P_{n}^{m}, Q_{n}^{m}$ are the Legendre functions of the first and second kinds, respectively. It is noted that $m, n$ must be integers $(m \leq n)$ in order that the Sturm-Liouville conditions are satisfied.

Since $\partial \varphi / \partial z=(1-2 v) \psi$

$$
\begin{aligned}
\varphi(\xi, \eta, \theta)= & \frac{a(1-2 v)}{\mathrm{i}(1+2 n)}\left[\frac{n+1-m}{n+1+m} Q_{n+1}^{m}(\mathrm{i} \xi)\right. \\
& \left.\times P_{n+1}^{m}(\eta)-\frac{n+m}{n-m} Q_{n-1}^{m}(\mathrm{i} \xi) P_{n-1}^{m}(\eta)\right] \\
& \times\left(b_{m n} \cos m \theta+c_{m n} \sin m \theta\right) .
\end{aligned}
$$

Substituting Eqs. (5) and (6) into Eq. (4), the displacements and the stresses can be obtained using the MATHEMATICA Software

$$
\begin{aligned}
u_{x} & =\left(b_{m n} u_{x c}^{m n}+c_{m n} u_{x s}^{m n}\right), \\
u_{y} & =\left(b_{m n} u_{y c}^{m n}+c_{m n} u_{y s}^{m n}\right), \\
u_{z} & =\left(b_{m n} u_{z c}^{m n}+c_{m n} u_{z s}^{m n}\right), \\
\sigma_{x x} & =\left(b_{m n} \sigma_{x x c}^{m n}+c_{m n} \sigma_{x x s}^{m n}\right), \\
\sigma_{y y} & =\left(b_{m n} \sigma_{y y c}^{m n}+c_{m n} \sigma_{y y s}^{m n}\right), \\
\sigma_{z z} & =\left(b_{m n} \sigma_{z z c}^{m n}+c_{m n} \sigma_{z z s}^{m n}\right), \\
\sigma_{x y} & =\left(b_{m n} \sigma_{x y c}^{m n}+c_{m n} \sigma_{x y s}^{m n}\right), \\
\sigma_{y z} & =\left(b_{m n} \sigma_{y z c}^{m n}+c_{m n} \sigma_{y z s}^{m n}\right), \\
\sigma_{x z} & =\left(b_{m n} \sigma_{x z c}^{m n}+c_{m n} \sigma_{x z s}^{m n}\right),
\end{aligned}
$$

where $u_{x c}^{m n}, u_{x s}^{m n}, \ldots, \sigma_{x x c}^{m n}, \sigma_{x x s}^{m n}, \ldots$ are the functions of $\xi, \eta, \theta, m, n$. These expressions are quite lengthy, the detailed formulas are not listed in this paper due to the space limitation.
For a single interior crack subject to symmetric crack face normal loading, the boundary conditions are

$$
\begin{aligned}
\sigma_{y z}(x, y, 0) & =\sigma_{x z}(x, y, 0)=0, \\
\sigma_{z z}(x, y, 0) & =p(x, y) \quad(\xi=0, \quad r<a), \\
u_{z}(x, y, 0) & =0 \quad(\eta=0, \quad r>a) .
\end{aligned}
$$

According to Eq. (4), we have

$\sigma_{x z}=\sigma_{y z}=0, \quad$ when $z=0$.

Equation (8a) is satisfied automatically. When $z=0$, we find

$u_{z}=\left\{\begin{array}{l}\frac{1-v}{\mu} Q_{n}^{m}(\mathrm{i} 0) P_{n}^{m}(\eta)\left(b_{m n} \cos m \theta+c_{m n} \sin m \theta\right) \\ \text { if } \xi=0(r<a), \\ \frac{1-v}{\mu} Q_{n}^{m}(\mathrm{i} \xi) P_{n}^{m}(0)\left(b_{m n} \cos m \theta+c_{m n} \sin m \theta\right) \\ \text { if } \eta=0(r>a) .\end{array}\right.$

If $m, n$ take opposite signs, $\left.P_{n}^{m}(\eta)\right|_{\eta=0}=0$, so that

$u_{z}=\left\{\begin{array}{l}\frac{1-v}{\mu} Q_{n}^{m}(\mathrm{i} 0) P_{n}^{m}(\eta)\left(b_{m n} \cos m \theta+c_{m n} \sin m \theta\right) \\ \quad \text { if } \xi=0(r<a), \\ 0 \quad \text { if } \eta=0(r>a) .\end{array}\right.$

One can easily show that

$\frac{\partial}{\partial z}=\frac{1}{a\left(\xi^{2}+\eta^{2}\right)}\left[\eta\left(1+\xi^{2}\right) \frac{\partial}{\partial \xi}+\xi\left(1-\eta^{2}\right) \frac{\partial}{\partial \eta}\right]$.

For $z=0$,

$\frac{\partial}{\partial z}= \begin{cases}\frac{1}{a \eta} \frac{\partial}{\partial \xi} & \text { if } \xi=0(r<a), \\ \frac{1}{a \xi} \frac{\partial}{\partial \eta} & \text { if } \eta=0(r>a) .\end{cases}$

Then,

$$
\begin{aligned}
\sigma_{z z}= & \frac{\partial \psi}{\partial z}-z \frac{\partial^{2} \psi}{\partial z^{2}} \\
= & \left\{\begin{array}{l}
\frac{1}{a \eta} \frac{\mathrm{d}}{\mathrm{d} \xi} Q_{n}^{m}(\mathrm{i} 0) P_{n}^{m}(\eta)\left(b_{m n} \cos m \theta+c_{m n} \sin m \theta\right) \\
\text { if } \xi=0(r<a) \\
\frac{1}{a \xi} Q_{n}^{m}(\mathrm{i} \xi) \frac{\mathrm{d}}{\mathrm{d} \eta} P_{n}^{m}(0)\left(b_{m n} \cos m \theta+c_{m n} \sin m \theta\right) \\
\text { if } \eta=0(r>a)
\end{array}\right.
\end{aligned}
$$

2.1.1.2 Shear loading According to the work of Kassir and Sih [22], by setting $\varphi_{1}=\partial \psi_{1} / \partial z, \varphi_{2}=\partial \psi_{2} / \partial z, \varphi_{3}=$ $G / 2, G=2\left(\partial \psi_{1} / \partial x+\partial \psi_{2} / \partial y\right), \partial G / \partial z=2\left(\partial \varphi_{1} / \partial x+\right.$ $\left.\partial \varphi_{2} / \partial y\right), F=z G$ in Eq. (3), the corresponding displacements and stresses are given by the following expressions 


$$
\begin{aligned}
& u_{x}=\frac{1}{2 \mu}\left[4(1-v) \varphi_{1}-z \frac{\partial G}{\partial x}\right], \\
& u_{y}=\frac{1}{2 \mu}\left[4(1-v) \varphi_{2}-z \frac{\partial G}{\partial y}\right], \\
& u_{z}=\frac{1}{2 \mu}\left[(1-2 v) G-z \frac{\partial G}{\partial z}\right], \\
& \sigma_{x x}=2 v \frac{\partial G}{\partial z}+4(1-v) \frac{\partial \varphi_{1}}{\partial x}-z \frac{\partial^{2} G}{\partial x^{2}}, \\
& \sigma_{y y}=2 v \frac{\partial G}{\partial z}+4(1-v) \frac{\partial \varphi_{2}}{\partial y}-z \frac{\partial^{2} G}{\partial y^{2}}, \\
& \sigma_{z z}=-z \frac{\partial^{2} G}{\partial z^{2}}, \\
& \sigma_{x y}=2(1-v) \frac{\partial \varphi_{1}}{\partial y}+2(1-v) \frac{\partial \varphi_{2}}{\partial x}-z \frac{\partial^{2} G}{\partial x \partial y}, \\
& \sigma_{y z}=2(1-v) \frac{\partial \varphi_{2}}{\partial z}-v \frac{\partial G}{\partial y}-z \frac{\partial^{2} G}{\partial y \partial z}, \\
& \sigma_{x z}=2(1-v) \frac{\partial \varphi_{1}}{\partial z}-v \frac{\partial G}{\partial x}-z \frac{\partial^{2} G}{\partial x \partial z} .
\end{aligned}
$$

In the oblate spheroidal coordinates, because $\varphi_{1}$ and $\varphi_{2}$ are harmonic functions which satisfy Laplace's equation (see 44), they take the form

$\varphi_{1}(\xi, \eta, \theta)=Q_{n}^{m}(\mathrm{i} \xi) P_{n}^{m}(\eta)\left(d_{m n} \cos m \theta+e_{m n} \sin m \theta\right)$,

$\varphi_{2}(\xi, \eta, \theta)=Q_{n}^{m}(\mathrm{i} \xi) P_{n}^{m}(\eta)\left(f_{m n} \cos m \theta+g_{m n} \sin m \theta\right)$.

Since $\varphi_{1}=\partial \psi_{1} / \partial z, \varphi_{2}=\partial \psi_{2} / \partial z$

$$
\begin{aligned}
\psi_{1}(\xi, \eta, \theta)= & \frac{a(1-2 v)}{\mathrm{i}(1+2 n)}\left[\frac{n+1-m}{n+1+m} Q_{n+1}^{m}(\mathrm{i} \xi) P_{n+1}^{m}(\eta)\right. \\
& \left.-\frac{n+m}{n-m} Q_{n-1}^{m}(\mathrm{i} \xi) P_{n-1}^{m}(\eta)\right] \\
& \times\left(d_{m n} \cos m \theta+e_{m n} \sin m \theta\right), \\
\psi_{2}(\xi, \eta, \theta)= & \frac{a(1-2 v)}{\mathrm{i}(1+2 n)}\left[\frac{n+1-m}{n+1+m} Q_{n+1}^{m}(\mathrm{i} \xi) P_{n+1}^{m}(\eta)\right. \\
& \left.-\frac{n+m}{n-m} Q_{n-1}^{m}(\mathrm{i} \xi) P_{n-1}^{m}(\eta)\right] \\
& \times\left(f_{m n} \cos m \theta+g_{m n} \sin m \theta\right) .
\end{aligned}
$$

Since $G=2\left(\partial \psi_{1} / \partial x+\partial \psi_{2} / \partial y\right)$, we have

$$
G=\left(d_{m n} G_{1 c}^{m n}+e_{m n} G_{1 s}^{m n}\right)+\left(f_{m n} G_{2 c}^{m n}+g_{m n} G_{2 s}^{m n}\right),
$$

where $G_{1 c}^{m n}, G_{1 s}^{m n}, G_{2 c}^{m n}$ and $G_{2 s}^{m n}$ are related with $\xi, \eta, \theta, m, n$.

Substituting Eqs. (15)-(19) to Eq. (14), using the MATHEMATICA Software, the components of the displacements and the stresses can be expressed as

$$
\begin{aligned}
u_{x} & =\left(d_{m n} u_{x 1 c}^{m n}+e_{m n} u_{x 1 s}^{m n}\right)+\left(f_{m n} u_{x 2 c}^{m n}+g_{m n} u_{x 2 s}^{m n}\right), \\
u_{y} & =\left(d_{m n} u_{y 1 c}^{m n}+e_{m n} u_{y 1 s}^{m n}\right)+\left(f_{m n} u_{y 2 c}^{m n}+g_{m n} u_{y 2 s}^{m n}\right),(20 \mathrm{a}) \\
u_{z} & =\left(d_{m n} u_{z 1 c}^{m n}+e_{m n} u_{z 1 s}^{m n}\right)+\left(f_{m n} u_{z 2 c}^{m n}+g_{m n} u_{z 2 s}^{m n}\right), \\
\sigma_{x x} & =\left(d_{m n} \sigma_{x x 1 c}^{m n}+e_{m n} \sigma_{x x 1 s}^{m n}\right)+\left(f_{m n} \sigma_{x x 2 c}^{m n}+g_{m n} \sigma_{x x 2 s}^{m n}\right), \\
\sigma_{y y} & =\left(d_{m n} \sigma_{y y 1 c}^{m n}+e_{m n} \sigma_{y y 1 s}^{m n}\right)+\left(f_{m n} \sigma_{y y 2 c}^{m n}+g_{m n} \sigma_{y y 2 s}^{m n}\right), \\
\sigma_{z z} & =\left(d_{m n} \sigma_{z z 1 c}^{m n}+e_{m n} \sigma_{z z 1 s}^{m n}\right)+\left(f_{m n} \sigma_{z z 2 c}^{m n}+g_{m n} \sigma_{z z 2 s}^{m n}\right), \\
\sigma_{x y} & =\left(d_{m n} \sigma_{x y 1 c}^{m n}+e_{m n} \sigma_{x y 1 s}^{m n}\right)+\left(f_{m n} \sigma_{x y 2 c}^{m n}+g_{m n} \sigma_{x y 2 s}^{m n}\right), \\
\sigma_{y z} & =\left(d_{m n} \sigma_{y z 1 c}^{m n}+e_{m n} \sigma_{y z 1 s}^{m n}\right)+\left(f_{m n} \sigma_{y z 2 c}^{m n}+g_{m n} \sigma_{y z 2 s}^{m n}\right), \\
\sigma_{x z} & =\left(d_{m n} \sigma_{x z 1 c}^{m n}+e_{m n} \sigma_{x z 1 s}^{m n}\right)+\left(f_{m n} \sigma_{x z 2 c}^{m n}+g_{m n} \sigma_{x z 2 s}^{m n}\right),
\end{aligned}
$$

where $u_{x 1 c}^{m n}, u_{x 1 s}^{m n}, \ldots, \sigma_{x x 1 c}^{m n}, \sigma_{x x 1 s}^{m n}, \ldots$ are functions of $\xi, \eta, \theta, m, n$, which are lengthy and will not be reproduced here.

For a single interior crack subject to antisymmetric crack face shear loading, the boundary conditions are

$$
\begin{aligned}
& \sigma_{z z}=0 \quad(|x|<\infty,|y|<\infty), \\
& \sigma_{x z}(x, y, 0)=q_{x}(x, y) \quad(\xi=0, r<a), \\
& \sigma_{y z}(x, y, 0)=q_{y}(x, y) \quad(\xi=0, r<a), \\
& u_{x}(x, y, 0)=u_{y}(x, y, 0)=0 \quad(\eta=0, r>a) .
\end{aligned}
$$

According to Eq. (14), we have

$\sigma_{z z}=0 \quad$ when $z=0$.

When $z=0$

$u_{x}=\left\{\begin{array}{cc}\frac{2(1-v)}{\mu} Q_{n}^{m}(\mathrm{i} 0) P_{n}^{m}(\eta) & \\ \times\left(d_{m n} \cos m \theta+e_{m n} \sin m \theta\right) & \text { if } \xi=0(r<a), \\ \frac{2(1-v)}{\mu} Q_{n}^{m}(\mathrm{i} \xi) P_{n}^{m}(0) & \\ \times\left(d_{m n} \cos m \theta+e_{m n} \sin m \theta\right) & \text { if } \eta=0(r>a) .\end{array}\right.$

If $m, n$ take opposite signs, $\left.P_{n}^{m}(\eta)\right|_{\eta=0}=0$, so that

$$
u_{x}=\left\{\begin{array}{l}
\frac{2(1-v)}{\mu} Q_{n}^{m}(\mathrm{i} 0) P_{n}^{m}(\eta) \\
\times\left(d_{m n} \cos m \theta+e_{m n} \sin m \theta\right) \quad \text { if } \xi=0(r<a), \\
0 \quad \text { if } \eta=0(r>a),
\end{array}\right.
$$$$
u_{y}=\left\{\begin{array}{l}
\frac{2(1-v)}{\mu} Q_{n}^{m}(\mathrm{i} 0) P_{n}^{m}(\eta) \\
\times\left(f_{m n} \cos m \theta+g_{m n} \sin m \theta\right) \quad \text { if } \xi=0(r<a), \\
0 \quad \text { if } \eta=0(r>a) .
\end{array}\right.
$$ 


\subsubsection{A set of arbitrary cracks}

For a linear elastic solid with $N$ cracks under remote loading $\sigma^{0}$, the problem can be decomposed into two problems. The first problem is a homogeneous problem, in which a homogeneous solid is subjected to remote load $\sigma^{0}$. The second problem can further be represented as a superposition of $N$ subproblems. Each subproblem is a single crack problem loaded by unknown opening and sliding displacements on the crack surface and the stresses are vanished at infinity. Based on superposition technique, by using the traction free conditions on each crack surface, a set of governing equation is formulated.

A global Cartesian coordinate system $(O x y z)$ is used, together with a local normal-tangential coordinate system with origin $\left(O_{k}\right)$ at the center of the $k$-th crack, represented by $x_{o k}, y_{o k}$ and $z_{o k}$. We let axis $z_{k}$ be the crack's normal direction and $y_{k}$ lie in the $x-y$ parallel plane. The orientation of the $k$-th crack is specified by the angle coordinates $\left(\alpha_{k}, \gamma_{k}\right)$ (see Fig. 1), where $0 \leq \gamma_{k} \leq \pi / 2$, $0 \leq \alpha_{k} \leq \pi$.

The linear elastic solid is subject to remote uniform loading $\sigma^{0}$, so the traction along the $k$-th crack surface produced by $\sigma^{0}$ is

$\boldsymbol{t}_{k}^{(0)}=\boldsymbol{n}_{k} \cdot \sigma^{0}$,

$p_{k}^{(0)}=\boldsymbol{n}_{k} \cdot \sigma^{0} \cdot \boldsymbol{n}_{k}$ and $\tau_{k}^{(0)}=\boldsymbol{n}_{k} \cdot \sigma^{0} \cdot\left(\boldsymbol{I}-\boldsymbol{n}_{k} \boldsymbol{n}_{k}\right)$ are the normal and shear tractions on the $k$-th crack induced by $\sigma^{0}$, where $\boldsymbol{n}_{k}$ is the unit normal vector on the $k$-th crack surface, $\boldsymbol{n}_{k} \boldsymbol{n}_{k}$ is a dyadic product.

As mentioned in Sect. 2.1.1, the displacement functions $\psi, \varphi, \varphi_{1}, \varphi_{2}, \psi_{1}$ and $\psi_{2}$ and the corresponding stresses $\sigma_{i j k}\left(\xi_{k}, \eta_{k}, \theta_{k}\right)(i, j=x, y, z)$ related with the $k$-th

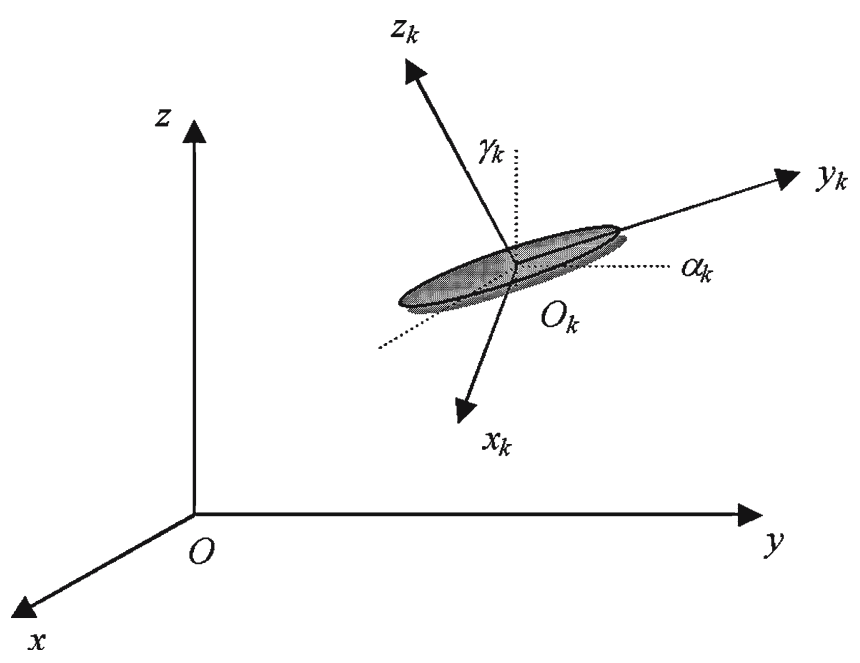

Fig. 1 The global and local coordinate system crack can be expressed in Legendre polynomial series for the normal and shear loading in the local coordinate system.

According to the formulae of coordinate system transformation, the tractions along the $l$-th crack surface in the local coordinate system $\left(O_{l} x_{l} y_{l} z_{l}\right)$ produced by the $k$-th crack can be written as follows

$\sigma_{i j l}^{(k)}=\boldsymbol{\beta}_{k l}^{\mathrm{T}} \sigma_{i j k} \boldsymbol{\beta}_{k l}$,

where $\boldsymbol{\beta}_{k l}$ is the matrix of coordinate system transformation between $O_{k} x_{k} y_{k} z_{k}$ and $O_{l} x_{l} y_{l} z_{l}$

$\boldsymbol{\beta}_{k l}=\boldsymbol{\beta}_{k}^{\mathrm{T}} \boldsymbol{\beta}_{l}$,

where $\boldsymbol{\beta}_{k}$ is the matrix of coordinate system transformation between $O x y z$ and $O_{k} x_{k} y_{k} z_{k}$.

$\boldsymbol{\beta}_{k}=\left[\begin{array}{lll}\cos \gamma_{k} \cos \alpha_{k}-\sin \alpha_{k} & \sin \gamma_{k} \cos \alpha_{k} \\ \cos \gamma_{k} \sin \alpha_{k} & \cos \alpha_{k} & \sin \gamma_{k} \sin \alpha_{k} \\ -\sin \gamma_{k} & 0 & \cos \gamma_{k}\end{array}\right]$.

According to the superposition scheme, the tractionfree conditions on each crack surface can be written as follows

$$
\begin{aligned}
& \sigma_{z z l}^{(0)}\left(0, \eta_{l}, \theta_{l}\right)+\sum_{k=1}^{N} \sigma_{z z l}^{(k)}\left(0, \eta_{l}, \theta_{l}\right)=0, \\
& \sigma_{x z l}^{(0)}\left(0, \eta_{l}, \theta_{l}\right)+\sum_{k=1}^{N} \sigma_{x z l}^{(k)}\left(0, \eta_{l}, \theta_{l}\right)=0, \\
& \sigma_{y z l}^{(0)}\left(0, \eta_{l}, \theta_{l}\right)+\sum_{k=1}^{N} \sigma_{y z l}^{(k)}\left(0, \eta_{l}, \theta_{l}\right)=0, \quad(l=1,2, \ldots, N),
\end{aligned}
$$

where $\sigma_{z z l}^{(0)}\left(0, \eta_{l}, \theta_{l}\right), \sigma_{x z l}^{(0)}\left(0, \eta_{l}, \theta_{l}\right)$ and $\sigma_{y z l}^{(0)}\left(0, \eta_{l}, \theta_{l}\right)$ are the tractions along the $l$-th crack surface in the local coordinate system $O_{l} x_{l} y_{l}\left(\xi_{l}, \eta_{l}, \theta_{l}\right)$ produced by the remote loading. $\sigma_{z z l}^{(k)}\left(0, \eta_{l}, \theta_{l}\right), \sigma_{x z l}^{(k)}\left(0, \eta_{l}, \theta_{l}\right)$ and $\sigma_{y z l}^{(k)}$ $\left(0, \eta_{l}, \theta_{l}\right)$ are the tractions along the $l$-th crack surface in the local coordinate system $\left(O_{l} x_{l} y_{l} z_{l}\left(\xi_{l}, \eta_{l}, \theta_{l}\right)\right)$ produced by the opening displacement loading and the sliding displacement loading on the $k$-th crack surface, respectively. Thus, Eqs. (29) are the governing equations for determining the unknown coefficients $b_{m n}^{k}, c_{m n}^{k}, d_{m n}^{k}$, $e_{m n}^{k}, f_{m n}^{k}, g_{m n}^{k}(k=1, \ldots, N ; m, n=0,1, \ldots, \infty)$.

\subsection{Calculation procedure}

\subsubsection{Boundary collocation method}

The governing equations are solved numerically on the basis of the crack surface boundary collocation method. By dividing the $k$-th crack surface into $C_{k}$ and $R_{k}$ elements in circumferential and axial direction, respectively, 
the collocation points on the $k$-th crack surface are given by the following expressions

$\xi_{k}(i, j)=0$,

$\eta_{k}(i, j)=\sqrt{1-\left(\frac{i}{R_{k}}\right)^{2}}$,

$\theta_{k}(i, j)=\frac{\pi}{C_{k}} j, \quad\left(i=1,2, \cdots, R_{k} ; j=1,2, \cdots, C_{k}\right)$.

The infinite series can be approximated with a sufficient degree of accuracy by the corresponding truncated series. The governing Eqs. (29) are reduced to a system of linear algebraic equations for the unknown coefficients $b_{m n}^{k}, c_{m n}^{k}, d_{m n}^{k}, e_{m n}^{k}, f_{m n}^{k}$ and $g_{m n}^{k}$.

When the algebraic equations are solved, the displacement functions and the stress components produced by each crack are known. According to the superposition principle, the stress fields produced by the multiple cracks are obtained through the transformation formulas from the local coordinate systems into the global one.

The stress intensity factors can be expressed as

$$
\begin{aligned}
& K_{\mathrm{I}}\left(\theta_{l}\right)=\lim _{\xi_{l} \rightarrow 0}\left\{\left(a_{l} \sqrt{1+\xi_{l}^{2}}-a_{l}\right)^{1 / 2} \sigma_{z z l}^{*}\left(\xi_{l}, 0, \theta_{l}\right)\right\}, \\
& K_{\mathrm{II}}\left(\theta_{l}\right)+\mathrm{i} K_{\mathrm{III}}\left(\theta_{l}\right)=\lim _{\xi_{l} \rightarrow 0}\left\{\left(a_{l} \sqrt{1+\xi_{l}^{2}}-a_{l}\right)^{1 / 2}\right. \\
& \times {\left.\left[\sigma_{x z l}^{*}\left(\xi_{l}, 0, \theta_{l}\right)+\mathrm{i} \sigma_{y z l}^{*}\left(\xi_{l}, 0, \theta_{l}\right)\right] \mathrm{e}^{-\mathrm{i} \theta_{l}}\right\}, }
\end{aligned}
$$

where $\sigma_{z z l}^{*}\left(\xi_{l}, 0, \theta_{l}\right), \sigma_{x z l}^{*}\left(\xi_{l}, 0, \theta_{l}\right)$ and $\sigma_{y z l}^{*}\left(\xi_{l}, 0, \theta_{l}\right)$ are the pseudo-tractions produced only by the opening displacement loading and the sliding displacement loading on the $l$-th crack surface.

\subsubsection{Average method of crack-surface tractions}

The average method for crack-surface tractions is used to solve the governing equations by approximately satisfying the traction-free conditions on the crack surface. According to the superposition technique, the tractions on each crack surface can be written as follows

$$
\begin{aligned}
\sigma_{z z l}= & \sigma_{z z l}^{(0)}+\sum_{k}\left[\sigma_{z z c l}^{m n(k)} b_{m n}^{k}+\sigma_{z z s l}^{m n(k)} c_{m n}^{k}+\sigma_{z z 1 c l}^{m n(k)} d_{m n}^{k}\right. \\
& \left.+\sigma_{z z 1 s l}^{m n(k)} e_{m n}^{k}+\sigma_{z z 2 c l}^{m n(k)} f_{m n}^{k}+\sigma_{z z 2 s l}^{m n(k)} g_{m n}^{k}\right], \\
\sigma_{y z l}= & \sigma_{y z l}^{(0)}+\sum_{k}\left[\sigma_{y z c l}^{m n(k)} b_{m n}^{k}+\sigma_{y z s l}^{m n(k)} c_{m n}^{k}+\sigma_{y z 1 c l}^{m n(k)} d_{m n}^{k}\right. \\
& \left.+\sigma_{y z 1 s l}^{m n(k)} e_{m n}^{k}+\sigma_{y z 2 c l}^{m n(k)} f_{m n}^{k}+\sigma_{y z 2 s l}^{m n(k)} g_{m n}^{k}\right], \\
\sigma_{x z l}= & \sigma_{x z l}^{(0)}+\sum_{k}\left[\sigma_{x z c l}^{m n(k)} b_{m n}^{k}+\sigma_{x z s l}^{m n(k)} c_{m n}^{k}+\sigma_{x z 1 c l}^{m n(k)} d_{m n}^{k}\right. \\
& \left.+\sigma_{x z 1 s l}^{m n(k)} e_{m n}^{k}+\sigma_{x z 2 c l}^{m n(k)} f_{m n}^{k}+\sigma_{x z 2 s l}^{m n(k)} g_{m n}^{k}\right], \\
& (l=1,2, \ldots, N) \quad(\operatorname{sum} \text { over } m, n),
\end{aligned}
$$

and they can be further expressed in the vector form as

$$
\begin{aligned}
\boldsymbol{t}_{l}= & \boldsymbol{t}_{l}^{(0)}+\boldsymbol{C}_{l k}^{m n} b_{m n}^{k}+\boldsymbol{S}_{l k}^{m n} c_{m n}^{k} \\
& +\overline{\boldsymbol{C}}_{l k}^{m n} d_{m n}^{k}+\overline{\boldsymbol{S}}_{l k}^{m n} e_{m n}^{k}+\overline{\overline{\boldsymbol{C}}}_{l k}^{m n} f_{m n}^{k}+\overline{\overline{\boldsymbol{S}}}_{l k}^{m n} g_{m n}^{k}, \\
& (l=1,2, \ldots, N) \quad(\text { sum over } k, m \text { and } n),
\end{aligned}
$$

where $\boldsymbol{t}_{l}$ is the traction column vector and $\boldsymbol{t}_{l}=\left[\sigma_{x z l}, \sigma_{y z} l\right.$, $\left.\sigma_{z z l}\right]^{\mathrm{T}} \cdot \boldsymbol{t}_{l}^{(0)}$ is the traction along the $l$-th crack surface in the local coordinate system $O_{l} x_{l} y_{l}$ produced by the remote loading $\sigma^{0} . \boldsymbol{C}_{l k}^{m n}, \boldsymbol{S}_{l k}^{m n}, \overline{\boldsymbol{C}}_{l k}^{m n}, \overline{\boldsymbol{S}}_{l k}^{m n}, \overline{\overline{\boldsymbol{C}}}_{l k}^{m n}$ and $\overline{\overline{\boldsymbol{S}}}_{l k}^{m n}$ are the tractions along the $l$-th crack surface in the local coordinate system $\left(O_{l} x_{l} y_{l} z_{l}\right)$ produced by the opening displacement loading and the sliding displacement loading on the $k$-th crack surface, respectively.

When the first order average of crack-surface tractions is used to yield the traction-free condition on the crack surface, namely, $m=0, n=1$, the governing equation is

$$
\begin{gathered}
\left\langle\boldsymbol{t}_{l}\right\rangle=\left\langle\boldsymbol{t}_{l}^{(0)}\right\rangle+\left\langle\boldsymbol{C}_{l k}^{01}\right\rangle b_{01}^{k}+\left\langle\overline{\boldsymbol{C}}_{l k}^{01}\right\rangle d_{01}^{k}+\left\langle\overline{\overline{\boldsymbol{C}}}_{l k}^{01}\right\rangle f_{01}^{k}=0, \\
(l=1,2, \ldots, N),
\end{gathered}
$$

where symbol \langle\rangle characterizes the average on the cracksurface. Then, the governing equations are reduced to a system of linear algebraic equations for the unknown coefficients $b_{01}^{k}, d_{01}^{k}$ and $f_{01}^{k}$. By solving the algebraic equations, $b_{01}^{k}, d_{01}^{k}$ and $f_{01}^{k}$ can be obtained.

When the second order averages of crack-surface tractions are used to yield the traction-free condition on crack surface, namely, $m=0, n=1$ and $m=0, n=3$, the governing equations are 


$$
\begin{aligned}
\left\langle\boldsymbol{t}_{l}\right\rangle= & \left\langle\boldsymbol{t}_{l}^{(0)}\right\rangle+\left\langle\boldsymbol{C}_{l k}^{01}\right\rangle b_{01}^{k}+\left\langle\overline{\boldsymbol{C}}_{l k}^{01}\right\rangle d_{01}^{k}+\left\langle\overline{\overline{\boldsymbol{C}}}_{l k}^{01}\right\rangle f_{01}^{k} \\
& +\left\langle\boldsymbol{C}_{l k}^{03}\right\rangle b_{03}^{k}+\left\langle\overline{\boldsymbol{C}}_{l k}^{03}\right\rangle d_{03}^{k}+\left\langle\overline{\overline{\boldsymbol{C}}}_{l k}^{03}\right\rangle f_{03}^{k}=0, \\
\left\langle\boldsymbol{t}_{l} \cdot \rho\right\rangle= & \left\langle\boldsymbol{t}_{l}^{(0)} \cdot \rho\right\rangle+\left\langle\boldsymbol{C}_{l k}^{01} \cdot \rho\right\rangle b_{01}^{k} \\
& +\left\langle\overline{\boldsymbol{C}}_{l k}^{01} \cdot \rho\right\rangle d_{01}^{k}+\left\langle\overline{\overline{\boldsymbol{C}}}_{l k}^{01} \cdot \rho\right\rangle f_{01}^{k}+\left\langle\boldsymbol{C}_{l k}^{03} \cdot \rho\right\rangle b_{03}^{k} \\
& +\left\langle\overline{\boldsymbol{C}}_{l k}^{03} \cdot \rho\right\rangle d_{03}^{k}+\left\langle\overline{\overline{\boldsymbol{C}}}_{l k}^{03} \cdot \rho\right\rangle f_{03}^{k}=0,
\end{aligned}
$$

where $\rho$ is the polar coordinate on crack surface. Then, the governing equations are reduced to a system of $6 \mathrm{~N}$ scalar linear algebraic equations for the unknown coefficients $b_{01}^{k}, d_{01}^{k}, f_{01}^{k}, b_{03}^{k}, d_{03}^{k}$ and $f_{03}^{k}$. By solving the algebraic equations, $b_{01}^{k}, d_{01}^{k}, f_{01}^{k}, b_{03}^{k}, d_{03}^{k}$ and $f_{03}^{k}$ can be obtained. Further, the tractions on crack-surface and SIFs along the $k$-th crack edges can be calculated.

When the $q$-th order averages of crack-surface tractions are used to yield the traction-free condition on crack surface, namely, $m=0, n=1,3,5, \ldots, 2 q-1$, the governing equations are

$\left\langle\boldsymbol{t}_{l}\right\rangle=0$,

$\left\langle\boldsymbol{t}_{l} \cdot \rho\right\rangle=0$,

$\left\langle\boldsymbol{t}_{l} \cdot \rho^{2}\right\rangle=0$,

$\left\langle\boldsymbol{t}_{l} \cdot \rho^{q-1}\right\rangle=0$.

By solving the algebraic equations, $b_{0 n}^{k}, d_{0 n}^{k}, f_{0 n}^{k}(n=$ $1,3,5, \ldots, 2 q-1)$ can be obtained. Further, the tractions on crack-surface and SIFs along the $k$-th crack edges can be calculated.

This paper presents a general method to solve the interaction problem including the strong interactions of arbitrary circle cracks. The first order approximation method given by Kachanov and Laures (1989) can yield quite good results for strong interacting crack problems. Hence we only extend their results to the second, third and $q$-th order approximations. Meanwhile we only use the terms with $m=0, n=1,3,5, \ldots$ (while Kachanov's average method only includes $m=0$ and $n=1$ ). Obviously when some cracks are very close to each other, we should use more terms including $m=1,2,3, \ldots$ and $n=m+1, m+3, \ldots$. Then Eq. (38) should include other equations, for example, $\left\langle\boldsymbol{t}_{l} \cos (m \theta)\right\rangle=0$, $\left\langle\boldsymbol{t}_{l} \sin (m \theta)\right\rangle=0,\left\langle\boldsymbol{t}_{l} \rho \cos (m \theta)\right\rangle=0,\left\langle\boldsymbol{t}_{l} \rho \sin (m \theta)\right\rangle=0, \ldots$, $\left\langle\boldsymbol{t}_{l} \rho^{q-1} \cos (m \theta)\right\rangle=0,\left\langle\boldsymbol{t}_{l} \rho^{q-1} \sin (m \theta)\right\rangle=0$ and so on. Then the calculation cost will increase greatly.

From Eqs. (29) and (33), the tractions produced by the opening and sliding displacement loading on crack surface can be expressed as

$$
\begin{aligned}
& \sigma_{z z l}^{*}(\rho, \theta)=-\left\{\sigma_{z z l}^{(0)}+\sum_{k \neq l}\left[\sigma_{z z c l}^{m n(k)} b_{m n}^{k}+\sigma_{z z s l}^{m n(k)} c_{m n}^{k}\right.\right. \\
& +\sigma_{z z 1 c l}^{m n(k)} d_{m n}^{k}+\sigma_{z z 1 s l}^{m n(k)} e_{m n}^{k} \\
& \left.\left.+\sigma_{z z 2 c l}^{m n(k)} f_{m n}^{k}+\sigma_{z z 2 s l}^{m n(k)} g_{m n}^{k}\right]\right\}, \\
& \sigma_{y z l}^{*}(\rho, \theta)=-\left\{\sigma_{y z l}^{(0)}+\sum_{k \neq l}\left[\sigma_{y z c l}^{m n(k)} b_{m n}^{k}+\sigma_{y z s l}^{m n(k)} c_{m n}^{k}\right.\right. \\
& +\sigma_{y z 1 c l}^{m n(k)} d_{m n}^{k}+\sigma_{y z 1 s l}^{m n(k)} e_{m n}^{k} \\
& \left.\left.+\sigma_{y z 2 c l}^{m n(k)} f_{m n}^{k}+\sigma_{y z 2 s l}^{m n(k)} g_{m n}^{k}\right]\right\}, \\
& \sigma_{x z l}^{*}(\rho, \theta)=-\left\{\sigma_{x z l}^{(0)}+\sum_{k \neq l}\left[\sigma_{x z c l}^{m n(k)} b_{m n}^{k}+\sigma_{x z s l}^{m n(k)} c_{m n}^{k}\right.\right. \\
& +\sigma_{x z 1 c l}^{m n(k)} d_{m n}^{k}+\sigma_{x z 1 s l}^{m n(k)} e_{m n}^{k} \\
& \left.\left.+\sigma_{x z 2 c l}^{m n(k)} f_{m n}^{k}+\sigma_{x z 2 s l}^{m n(k)} g_{m n}^{k}\right]\right\}, \\
& (l=1,2, \cdots, N) \quad(\text { sum over } m, n) .
\end{aligned}
$$

The SIFs are given as (Kachanov and Laures [20] and Fabrikant [24])

$$
K_{\mathrm{I}}(\theta)=\frac{1}{\pi^{2} \sqrt{2 a}} \times \int_{0}^{2 \pi} \int_{0}^{a} \frac{\sqrt{a^{2}-\rho_{0}^{2}} \sigma_{z z}^{*}\left(\rho_{0}, \theta_{0}\right) \rho_{0} \mathrm{~d} \rho_{0} \mathrm{~d} \theta_{0}}{a^{2}+\rho_{0}^{2}-2 a \rho_{0} \cos \left(\theta-\theta_{0}\right)},
$$

$$
\begin{aligned}
K_{\mathrm{II}}(\theta)+\mathrm{i} K_{\mathrm{III}}(\theta)= & \frac{1}{\pi^{2} \sqrt{2 a}} \int_{0}^{2 \pi} \int_{0}^{a} \sqrt{a^{2}-\rho_{0}^{2}} \\
& \times\left\{\frac{\mathrm{e}^{-\mathrm{i} \theta} \tau^{*}\left(\rho_{0}, \theta_{0}\right)}{a^{2}+\rho_{0}^{2}-2 a \rho_{0} \cos \left(\theta-\theta_{0}\right)}\right. \\
& \left.+\frac{v}{2-v} \frac{\mathrm{e}^{\mathrm{i} \theta}\left(3 a-\rho_{0} \mathrm{e}^{\mathrm{i}\left(\theta-\theta_{0}\right)}\right) \overline{\tau^{*}}\left(\rho_{0}, \theta_{0}\right)}{a\left(a-\rho_{0} \mathrm{e}^{\mathrm{i}\left(\theta-\theta_{0}\right)}\right)^{2}}\right\} \\
& \times \rho_{0} \mathrm{~d} \rho_{0} \mathrm{~d} \theta_{0},
\end{aligned}
$$

where $\tau^{*}=\sigma_{x z}^{*}+\mathrm{i} \sigma_{y z}^{*}$, an overbar denotes a complex conjugate.

\section{Numerical examples}

\subsection{Two coplanar cracks under normal loading}

\subsubsection{Two coplanar cracks of equal size (Fig. 2)}

Firstly, the first, second, third and fourth order average traction methods on crack-surface are used to calculate the stress intensity factors along the edges of two 


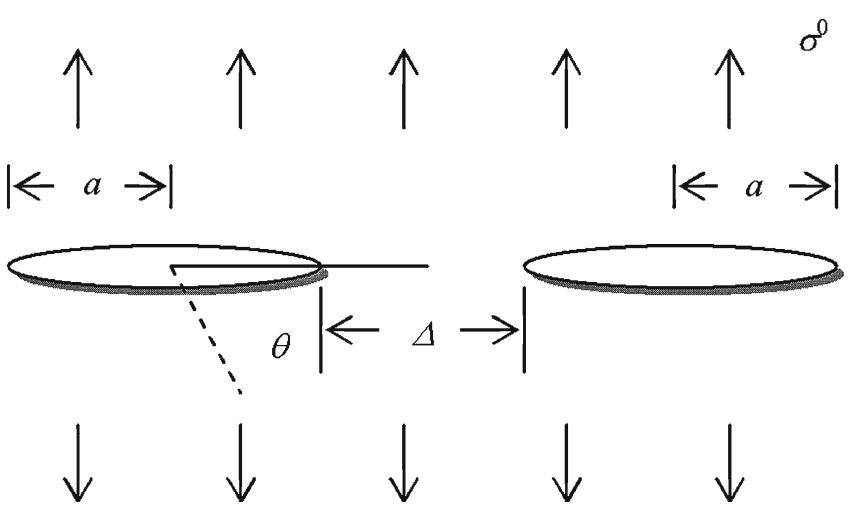

Fig. 2 Two coplanar cracks of equal size under normal loading

coplanar cracks of the same size under normal loading. The results are presented and compared with those of Kachanov and Laures [20] and Fabrikant [18] in Tables 1 and 2. As seen in the tables, the interaction produces a stress amplification, i.e., $K_{\mathrm{I}} / K_{\mathrm{I}}^{0}>1$ everywhere along the edge, which agrees with Kachanov's conclusion. The maximal error of $K_{\mathrm{I}} / K_{\mathrm{I}}^{0}$ between the present results and Kachanov's solutions is less than $0.2 \%$ when the first order average traction method is used, indicating that the present solutions are accurate enough for very closely spaced and strongly interacting cracks. The differences of the values of $K_{\mathrm{I}} / K_{\mathrm{I}}^{0}$ obtained by using the first and fourth order average traction methods are less than $0.5 \%$ at spacings $0.05<\Delta / 2 a<0.25$, which indicates that the accuracy of the first order average traction method is high enough. The differences increase as the spacing decreases and become $0.98 \%$ when $\Delta / 2 a=0.00025$.

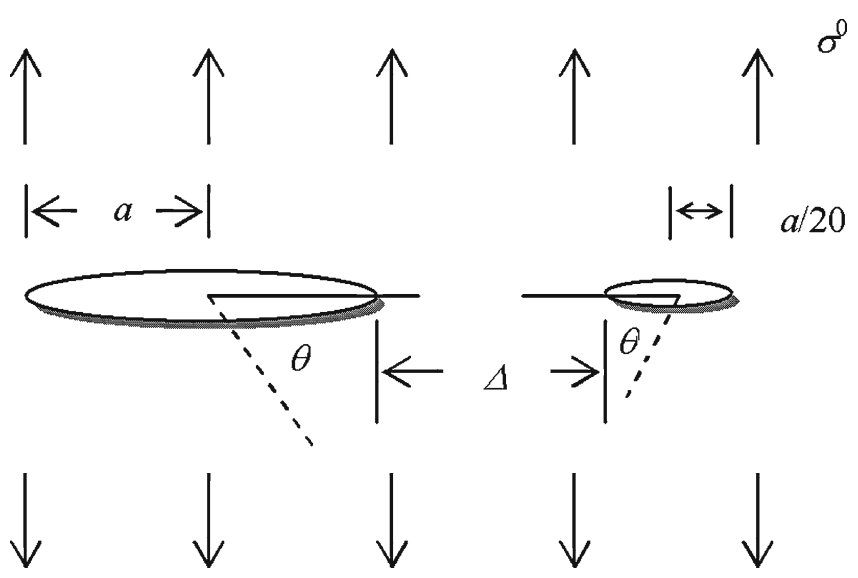

Fig. 3 Coplanar crack-microcrack interaction under normal loading

\subsubsection{Coplanar crack-microcrack interaction (Fig. 3)}

Stress intensity fractors for two coplanar crackmicrocrack interactions under normal loading are calculated by using the first and second order averages. The microcrack size is $1 / 20$ of the main crack size. The results are given in Tables 3 and 4, along with Kachanov's results (see e.g. Kachanov and Laures [20]). It is observed that the present results are in good agreement with Kachanov's solutions. When the first order average is employed, the maximal error of $K_{\mathrm{I}} / K_{\mathrm{I}}^{0}$ between the present results and Kachanov's solutions is less than $0.2 \%$. Effect of the small crack on the large one is quite slight except for the points that are closest to the small crack; effect of the large crack on the small one is quite significant at all points of the small crack edge.

\subsection{Two stacked cracks in normal loading}

\subsubsection{Two stacked cracks of the same size (Fig. 4)}

Stress intensity fractors of two stacked cracks of the same size in normal loading are calculated using the boundary collocation procedure. The results are presented in Table 5, along with the solutions of other methods (see e.g. Kachanov and Laures [20], Isida et al. [12], etc.). As shown in the table, the interaction results not

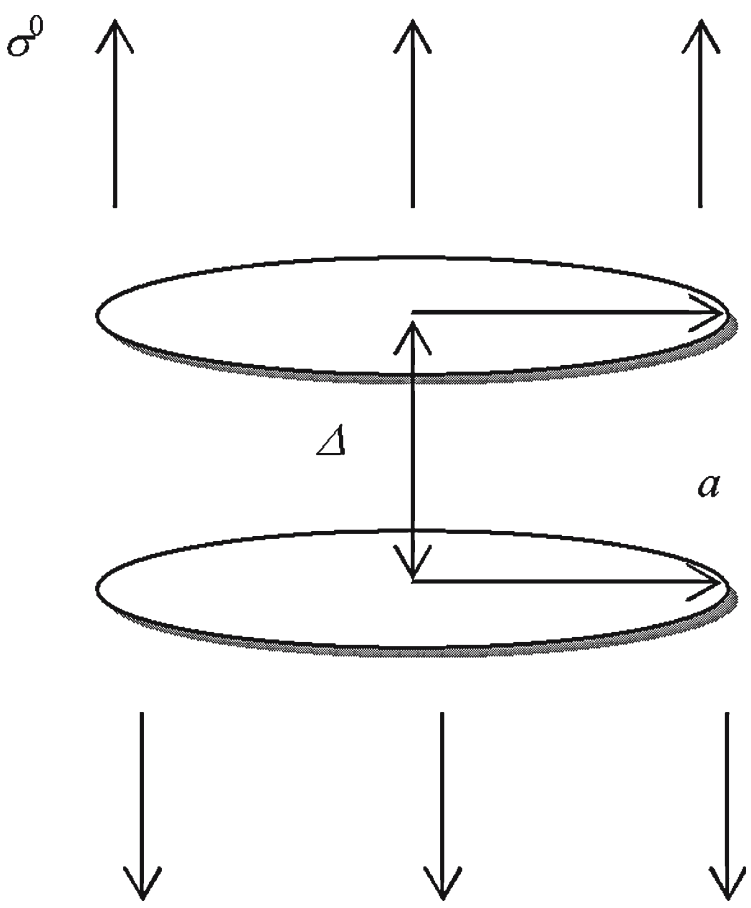

Fig. 4 Two stacked crack of equal size under normal loading 
Table 1 The values of max $\left(K_{\mathrm{I}} / K_{\mathrm{I}}^{0}\right)$ for two coplanar cracks of equal size under normal loading $(v=0.25)$
Table 2 Two coplanar closely spaced cracks of equal size under normal loading. The values of $K_{\mathrm{I}} / K_{\mathrm{I}}^{0}$ around the crack edge $(v=0.25)$

\begin{tabular}{lllllll}
\hline$\Delta / 2 a$ & Ref. [20] & Ref. [18] & \multicolumn{4}{c}{ Present results } \\
\cline { 4 - 6 } & & & First order & Second order & Third order & Fourth order \\
\hline 0.00025 & 2.8819 & 2.7758 & 2.87827 & 2.89272 & 2.90242 & 2.90653 \\
0.005 & 1.9078 & 1.86131 & 1.90505 & 1.911251 & 1.91234 & 1.91242 \\
0.05 & 1.2964 & 1.28777 & 1.29588 & 1.29564 & 1.29551 & 1.29547 \\
0.10 & 1.1711 & 1.16785 & 1.17083 & 1.17060 & 1.17055 & 1.17054 \\
0.15 & 1.1153 & & 1.11518 & 1.11504 & 1.11502 & 1.11502 \\
0.25 & 1.0639 & 1.06342 & 1.06380 & 1.06375 & 1.06374 & 1.06374 \\
0.50 & 1.0224 & & 1.02348 & 1.02347 & 1.02347 & 1.02347 \\
1.00 & 1.0067 & & 1.00670 & 1.00670 & 1.00670 & 1.00670 \\
1.50 & 1.0029 & & 1.00285 & 1.00285 & 1.00285 & 1.00285
\end{tabular}

\begin{tabular}{|c|c|c|c|c|c|c|c|c|}
\hline \multirow[t]{3}{*}{$\theta$} & \multicolumn{4}{|c|}{$\Delta / 2 a=0.05$} & \multicolumn{4}{|c|}{$\Delta / 2 a=0.00025$} \\
\hline & \multirow[t]{2}{*}{ Ref. [20] } & \multirow[t]{2}{*}{ Ref. [18] } & \multicolumn{2}{|c|}{ Present results } & \multirow[t]{2}{*}{ Ref. [20] } & \multirow[t]{2}{*}{ Ref. [18] } & \multicolumn{2}{|c|}{ Present results } \\
\hline & & & First order & Second order & & & First order & Second order \\
\hline 0 & 1.2964 & 1.2877 & 1.29588 & 1.29564 & 2.8819 & 2.7758 & 2.87827 & 2.89272 \\
\hline 15 & 1.2192 & 1.2131 & 1.21723 & 1.21712 & 1.4393 & 1.4161 & 1.43704 & 1.43745 \\
\hline 30 & 1.128 & 1.1255 & 1.12694 & 1.12651 & 1.1957 & 1.1863 & 1.19412 & 1.19257 \\
\hline 45 & 1.0791 & 1.0772 & 1.07745 & 1.07725 & 1.1091 & 1.1042 & 1.10752 & 1.10693 \\
\hline 60 & 1.0529 & 1.0518 & 1.05178 & 1.05146 & 1.0699 & 1.0669 & 1.06827 & 1.06769 \\
\hline 75 & 1.038 & 1.0375 & 1.03756 & 1.03736 & 1.0494 & 1.0473 & 1.04848 & 1.04798 \\
\hline 90 & 1.0296 & 1.0289 & 1.02889 & 1.02876 & 1.0374 & 1.0361 & 1.03680 & 1.03645 \\
\hline 105 & 1.0241 & 1.0236 & 1.02373 & 1.02356 & 1.0303 & 1.0291 & 1.02989 & 1.02960 \\
\hline 120 & 1.0205 & 1.0201 & 1.02020 & 1.02011 & 1.0257 & 1.0247 & 1.02538 & 1.02513 \\
\hline 135 & 1.0182 & 1.0179 & 1.01803 & 1.01794 & 1.0228 & 1.0219 & 1.02244 & 1.02221 \\
\hline 150 & 1.0168 & 1.0164 & 1.01654 & 1.01646 & 1.0209 & 1.0201 & 1.02065 & 1.02044 \\
\hline 165 & 1.0159 & 1.0156 & 1.01576 & 1.01568 & 1.0199 & 1.0191 & 1.01966 & 1.01945 \\
\hline 180 & 1.0157 & 1.0154 & 1.01544 & 1.01536 & 1.0196 & 1.0188 & 1.01926 & 1.01906 \\
\hline
\end{tabular}

Table 3 The values of max $K_{\mathrm{I}} / K_{\mathrm{I}}^{0}$ for coplanar crack-microcrack interaction under normal loading $(v=0.25)$

\begin{tabular}{|c|c|c|c|c|c|c|}
\hline \multirow[t]{3}{*}{$\Delta / 2 a$} & \multicolumn{2}{|c|}{ Ref. [20] } & \multicolumn{4}{|c|}{ Present results } \\
\hline & \multirow[b]{2}{*}{$\begin{array}{l}\text { Main crack } \\
\max \left(K_{\mathrm{I}} / K_{\mathrm{I}}^{0}\right)\end{array}$} & \multirow[b]{2}{*}{$\begin{array}{l}\text { Microcrack } \\
\max \left(K_{\mathrm{I}} / K_{\mathrm{I}}^{0}\right)\end{array}$} & \multicolumn{2}{|c|}{ First order } & \multicolumn{2}{|c|}{ Second order } \\
\hline & & & $\begin{array}{l}\text { Main crack } \\
\max \left(K_{\mathrm{I}} / K_{\mathrm{I}}^{0}\right)\end{array}$ & $\begin{array}{l}\text { Microcrack } \\
\max \left(K_{\mathrm{I}} / K_{\mathrm{I}}^{0}\right)\end{array}$ & $\begin{array}{l}\text { Main crack } \\
\max \left(K_{\mathrm{I}} / K_{\mathrm{I}}^{0}\right)\end{array}$ & $\begin{array}{l}\text { Microcrack } \\
\max \left(K_{\mathrm{I}} / K_{\mathrm{I}}^{0}\right)\end{array}$ \\
\hline 0.05 & 1.1662 & 4.0963 & 1.16431 & 4.08925 & 1.16420 & 4.09248 \\
\hline 0.10 & 1.0943 & 3.3527 & 1.09335 & 3.34883 & 1.09293 & 3.35082 \\
\hline 0.15 & 1.0625 & 2.9563 & 1.06199 & 2.95331 & 1.06171 & 2.95483 \\
\hline 0.20 & 1.0448 & 2.6947 & 1.04445 & 2.69218 & 1.04426 & 2.69334 \\
\hline 0.30 & 1.0262 & 2.3564 & 1.02605 & 2.35443 & 1.02597 & 2.35526 \\
\hline 0.40 & 1.0171 & 2.1394 & 1.01697 & 2.13784 & 1.01694 & 2.13849 \\
\hline 0.50 & 1.0119 & 1.9850 & 1.01182 & 1.98370 & 1.01180 & 1.98424 \\
\hline 1.00 & 1.0033 & 1.5856 & 1.00327 & 1.58489 & 1.00327 & 1.58518 \\
\hline 1.50 & 1.0014 & 1.4083 & 1.00139 & 1.40781 & 1.00139 & 1.40800 \\
\hline
\end{tabular}

only show a shielding of the mode I SIF but also see an appearance of mode II SIF, which agrees well with the conclusion of Kachanov. When the spacing $\Delta / 2 a$ between cracks is small, our results agree with Kachanov's results; and they are also in good agreement with Isida's solutions for large spacings. 
Table 4 Coplanar

crack-microcrack interaction under normal loading. The values of $K_{\mathrm{I}} / K_{\mathrm{I}}^{0}$ along the edges of the main crack and of the microcrack

$(\Delta / 2 a=0.01, v=0.25)$

\begin{tabular}{|c|c|c|c|c|c|c|}
\hline \multirow[t]{3}{*}{$\theta$} & \multicolumn{2}{|c|}{ Ref. [20] } & \multicolumn{4}{|c|}{ Present results } \\
\hline & \multirow[b]{2}{*}{$\begin{array}{l}\text { Main crack } \\
K_{\mathrm{I}} / K_{\mathrm{I}}^{0}\end{array}$} & \multirow[b]{2}{*}{$\begin{array}{l}\text { Microcrack } \\
K_{\mathrm{I}} / K_{\mathrm{I}}^{0}\end{array}$} & \multicolumn{2}{|c|}{ First order } & \multicolumn{2}{|c|}{ Second order } \\
\hline & & & $\begin{array}{l}\text { Main crack } \\
K_{\mathrm{I}} / K_{\mathrm{I}}^{0}\end{array}$ & $\begin{array}{l}\text { Microcrack } \\
K_{\mathrm{I}} / K_{\mathrm{I}}^{0}\end{array}$ & $\begin{array}{l}\text { Main crack } \\
K_{\mathrm{I}} / K_{\mathrm{I}}^{0}\end{array}$ & $\begin{array}{l}\text { Microcrack } \\
K_{\mathrm{I}} / K_{\mathrm{I}}^{0}\end{array}$ \\
\hline 0 & 1.0943 & 3.3527 & 1.09335 & 3.34883 & 1.09293 & 3.35082 \\
\hline 15 & 1.0024 & 3.2171 & 1.00234 & 3.21577 & 1.00230 & 3.21612 \\
\hline 30 & 1.0006 & 2.9260 & 1.00061 & 2.92456 & 1.00060 & 2.92484 \\
\hline 45 & 1.0003 & 2.6313 & 1.00028 & 2.62878 & 1.00028 & 2.62903 \\
\hline 60 & 1.0002 & 2.3876 & 1.00017 & 2.38690 & 1.00017 & 2.38711 \\
\hline 90 & 1.0001 & 2.0550 & 1.00008 & 2.05423 & 1.00008 & 2.05436 \\
\hline 120 & 1.0001 & 1.8679 & 1.00006 & 1.86687 & 1.00005 & 1.86709 \\
\hline 150 & 1.0000 & 1.7729 & 1.00003 & 1.77233 & 1.00003 & 1.77261 \\
\hline 180 & 1.0000 & 1.7438 & 1.00002 & 1.74362 & 1.00002 & 1.74374 \\
\hline
\end{tabular}

Table 5 The values of $K_{\mathrm{I}} / K_{\mathrm{I}}^{0}$ and $K_{\mathrm{II}} / K_{\mathrm{I}}^{0}$ for two stacked cracks of equal size under normal loading $(v=0.25)$

\begin{tabular}{lllllll}
\hline$\Delta / 2 a$ & \multicolumn{3}{c}{$K_{\mathrm{I}} / K_{\mathrm{I}}^{0}$} & & \multicolumn{2}{c}{$K_{\mathrm{II}} / K_{\mathrm{I}}^{0}$} \\
\cline { 2 - 3 } & Present results & Ref. [20] & Ref. [12] & & Present results & Ref. [12] \\
\hline 0.25 & 0.767776 & 0.7678 & 0.7759 & & 0.13816 & 0.1390 \\
0.35 & 0.795536 & 0.7898 & & 0.11729 & 0.0910 \\
0.50 & 0.831634 & 0.8249 & 0.8356 & 0.09028 & 0.0549 \\
0.75 & 0.881329 & 0.8781 & 0.8828 & & 0.05506 & 0.0325 \\
1.00 & 0.918728 & 0.9176 & 0.9189 & & 0.03220 & 0.0115 \\
1.50 & 0.961547 & 0.9614 & 0.9613 & & 0.01140 & \\
2.00 & 0.980182 & & 0.9802 & & \\
5.00 & 0.998381 & & 0.9990 & & \\
\hline
\end{tabular}

\subsubsection{Stacked crack-microcrack interaction (Fig. 5)}

$\sigma^{0}$
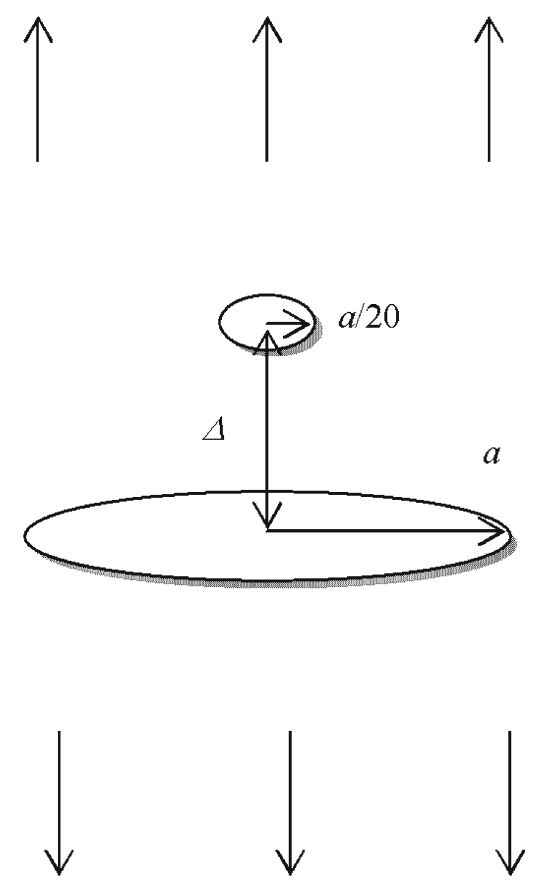

Fig. 5 Stacked crack-microcrack interaction under normal loading
The boundary collocation procedure is employed to analyze SIFs for stacked crack-microcrack interactions in normal loading. The microcrack size is $1 / 20$ of the main crack size. The present solutions are given and compared with Kachanov's results (see e.g. Kachanov and Laures [20]) in Table 6. Table 7 shows that the agreement is quite good. The value of $K_{\mathrm{I}} / K_{\mathrm{I}}^{0}$ for the main crack is equal to 1 indicating that the effect of the microcrack on the main crack is negligible; and microcrack's $K_{\mathrm{I}} / K_{\mathrm{I}}^{0}<1$, which shows the crack-microcrack interaction produces a shielding for the microcrack.

3.3 "V" arrangement under normal loading (Fig. 6) and " $\mathrm{H}$ " arrangement under shear (Fig. 7)

"V" arrangement under normal loading and "H" arrangement under shear are considered. SIFs are calculated by using the first order average traction method. All cracks have the same size for "V" and " $H$ " crack configuration, respectively. The present and Kachanov's results (Kachanov and Laures [20]) are given in Tables 7, 8 and 9, where $K_{\mathrm{I}}^{0}, K_{\mathrm{II}}^{0}$ and $K_{\mathrm{III}}^{0}$ are the stress intensity factors 
Table 6 The values of max $K_{\mathrm{I}} / K_{\mathrm{I}}^{0}$ for stacked crack-microcrack interaction under normal loading $(v=0.25)$

\begin{tabular}{|c|c|c|c|c|}
\hline \multirow[t]{2}{*}{$\Delta / 2 \mathrm{a}$} & \multicolumn{2}{|c|}{ Ref. [20] } & \multicolumn{2}{|c|}{ Present results } \\
\hline & $\begin{array}{l}\text { Main crack } \\
\max \left(K_{\mathrm{I}} / K_{\mathrm{I}}^{0}\right)\end{array}$ & $\begin{array}{l}\text { Microcrack } \\
\max \left(K_{\mathrm{I}} / K_{\mathrm{I}}^{0}\right)\end{array}$ & $\begin{array}{l}\text { Main crack } \\
\max \left(K_{\mathrm{I}} / K_{\mathrm{I}}^{0}\right)\end{array}$ & $\begin{array}{l}\text { Microcrack } \\
\max \left(K_{\mathrm{I}} / K_{\mathrm{I}}^{0}\right)\end{array}$ \\
\hline 0.0025 & 1.0000 & 0.0002 & 1.0000 & 0.00019 \\
\hline 0.05 & 1.0000 & 0.0017 & 1.0000 & 0.00167 \\
\hline 0.15 & 1.0000 & 0.0395 & 1.0000 & 0.03951 \\
\hline 0.25 & 1.0000 & 0.1430 & 1.0000 & 0.14307 \\
\hline 0.35 & 1.0000 & 0.2873 & 1.0000 & 0.28735 \\
\hline 0.45 & 1.0000 & 0.4341 & 1.0000 & 0.43419 \\
\hline 0.55 & 1.0000 & 0.5611 & 1.0000 & 0.56113 \\
\hline 0.65 & 1.0000 & 0.6621 & 1.0000 & 0.66203 \\
\hline 0.75 & 1.0000 & 0.7391 & 1.0000 & 0.73907 \\
\hline 1.00 & 1.0000 & 0.8578 & 1.0000 & 0.85779 \\
\hline
\end{tabular}

Table 7 The values of SIFs around the crack edge for "V" crack configuration $(v=0.25)$

\begin{tabular}{|c|c|c|c|c|c|c|c|c|c|c|c|c|}
\hline \multirow[t]{2}{*}{$\theta$} & \multicolumn{2}{|c|}{ Ref. [20] } & \multicolumn{2}{|c|}{ Present results } & \multicolumn{2}{|c|}{ Ref. [20] } & \multicolumn{2}{|c|}{ Present results } & \multicolumn{2}{|c|}{ Ref. [20] } & \multicolumn{2}{|c|}{ Present results } \\
\hline & $\frac{K_{\mathrm{I}}}{\sigma^{0} \sqrt{a}}$ & $\frac{K_{\mathrm{I}}^{0}}{\sigma^{0} \sqrt{a}}$ & $\frac{K_{\mathrm{I}}}{\sigma^{0} \sqrt{a}}$ & $\frac{K_{\mathrm{I}}^{0}}{\sigma^{0} \sqrt{a}}$ & $\frac{K_{\mathrm{II}}}{\sigma^{0} \sqrt{a}}$ & $\frac{K_{\mathrm{II}}^{0}}{\sigma^{0} \sqrt{a}}$ & $\frac{K_{\mathrm{II}}}{\sigma^{0} \sqrt{a}}$ & $\frac{K_{\mathrm{II}}^{0}}{\sigma^{0} \sqrt{a}}$ & $\frac{K_{\mathrm{III}}}{\sigma^{0} \sqrt{a}}$ & $\frac{K_{\mathrm{III}}^{0}}{\sigma^{0} \sqrt{a}}$ & $\frac{K_{\mathrm{III}}}{\sigma^{0} \sqrt{a}}$ & $\frac{K_{\mathrm{III}}^{0}}{\sigma^{0} \sqrt{a}}$ \\
\hline 0 & 0.3253 & 0.2251 & 0.32591 & 0.2251 & -0.2465 & -0.2572 & -0.24635 & -0.2572 & 0.0000 & 0.0000 & 0.00000 & 0.0000 \\
\hline 30 & 0.2765 & 0.2251 & 0.27704 & 0.2251 & -0.2765 & -0.2227 & -0.22628 & -0.2227 & 0.0837 & 0.0964 & 0.08402 & 0.0964 \\
\hline 60 & 0.2391 & 0.2251 & 0.23934 & 0.2251 & -0.1334 & -0.1286 & -0.13324 & -0.1286 & 0.1642 & 0.1670 & 0.16415 & 0.1670 \\
\hline 90 & 0.2284 & 0.2251 & 0.22883 & 0.2251 & 0.0020 & 0.0000 & 0.00154 & 0.0000 & 0.1961 & 0.1929 & 0.19584 & 0.1929 \\
\hline 120 & 0.2257 & 0.2251 & 0.22601 & 0.2251 & 0.1331 & 0.1286 & 0.13283 & 0.1286 & 0.1709 & 0.1671 & 0.17083 & 0.1671 \\
\hline 150 & 0.2250 & 0.2251 & 0.22523 & 0.2251 & 0.2299 & 0.2227 & 0.22957 & 0.2227 & 0.0987 & 0.0964 & 0.09856 & 0.0964 \\
\hline 180 & 0.2248 & 0.2251 & 0.22501 & 0.2251 & 0.2653 & 0.2572 & 0.26503 & 0.2572 & 0.0000 & 0.0000 & 0.00000 & 0.0000 \\
\hline
\end{tabular}

Table 8 The values of SIFs around the crack edge of crack 1 for " $H$ " crack configuration $(v=0.25)$

\begin{tabular}{|c|c|c|c|c|c|c|c|c|c|c|c|c|}
\hline \multirow[t]{2}{*}{$\theta$} & \multicolumn{2}{|c|}{ Ref. [20] } & \multicolumn{2}{|c|}{ Present results } & \multicolumn{2}{|c|}{ Ref. [20] } & \multicolumn{2}{|c|}{ Present results } & \multicolumn{2}{|c|}{ Ref. [20] } & \multicolumn{2}{|c|}{ Present results } \\
\hline & $\frac{K_{\mathrm{I}}}{\tau^{0} \sqrt{a}}$ & $\frac{K_{\mathrm{I}}^{0}}{\tau^{0} \sqrt{a}}$ & $\frac{K_{\mathrm{I}}}{\tau^{0} \sqrt{a}}$ & $\frac{K_{\mathrm{I}}^{0}}{\tau^{0} \sqrt{a}}$ & $\frac{K_{\mathrm{II}}}{\tau^{0} \sqrt{a}}$ & $\frac{K_{\mathrm{II}}^{0}}{\tau^{0} \sqrt{a}}$ & $\frac{K_{\mathrm{II}}}{\tau^{0} \sqrt{a}}$ & $\frac{K_{\mathrm{II}}^{0}}{\tau^{0} \sqrt{a}}$ & $\frac{K_{\mathrm{III}}}{\tau^{0} \sqrt{a}}$ & $\frac{K_{\mathrm{III}}^{0}}{\tau^{0} \sqrt{a}}$ & $\frac{K_{\mathrm{III}}}{\tau^{0} \sqrt{a}}$ & $\frac{K_{\mathrm{III}}^{0}}{\tau^{0} \sqrt{a}}$ \\
\hline 0 & -0.0704 & 0.0000 & -0.07038 & 0.0000 & 0.4719 & 0.5144 & 0.47192 & 0.51447 & 0.0000 & 0.0000 & 0.00000 & 0.00000 \\
\hline 30 & -0.0716 & 0.0000 & -0.07159 & 0.0000 & 0.4043 & 4455 & 0.40482 & 44554 & -0.1923 & -0.1929 & -0.19255 & -0.19292 \\
\hline 60 & -0.0674 & 0.0000 & -0.06726 & 0.0000 & 0.2241 & 0.2572 & 0.22461 & 0.25723 & -0.3434 & -0.3341 & -0.34303 & -0.33416 \\
\hline 90 & 0.0000 & 0.0000 & 0.00000 & 0.0000 & 0.0000 & 0.0000 & 0.00000 & 0.00000 & -0.4187 & -0.3858 & -0.41864 & -0.38585 \\
\hline 120 & 0.0674 & 0.0000 & 0.06726 & 0.0000 & -0.2241 & -0.2572 & -0.22461 & -0.25723 & -0.3434 & -0.3341 & -0.34303 & -0.33416 \\
\hline 150 & 0.0716 & 0.0000 & 0.07159 & 0.0000 & -0.4042 & -0.4455 & -0.40482 & -0.44554 & -0.1923 & -0.1929 & -0.19255 & -0.19292 \\
\hline 180 & 0.0704 & 0.0000 & 0.07038 & 0.0000 & -0.4719 & -0.5144 & -0.47192 & -0.51447 & 0.0000 & 0.0000 & 0.00000 & 0.00000 \\
\hline
\end{tabular}

Table 9 The values of SIFs around the crack edge of crack 2 for " $\mathrm{H}$ " crack configuration $(v=0.25)$

\begin{tabular}{|c|c|c|c|c|c|c|c|c|c|c|c|c|}
\hline \multirow[t]{2}{*}{$\theta$} & \multicolumn{2}{|c|}{ Ref. [20] } & \multicolumn{2}{|c|}{ Present results } & \multicolumn{2}{|c|}{ Ref. [20] } & \multicolumn{2}{|c|}{ Present results } & \multicolumn{2}{|c|}{ Ref. [20] } & \multicolumn{2}{|c|}{ Present results } \\
\hline & $\frac{K_{\mathrm{I}}}{\tau^{0} \sqrt{a}}$ & $\frac{K_{\mathrm{I}}^{0}}{\tau^{0} \sqrt{a}}$ & $\frac{K_{\mathrm{I}}}{\tau^{0} \sqrt{a}}$ & $\frac{K_{\mathrm{I}}^{0}}{\tau^{0} \sqrt{a}}$ & $\frac{K_{\mathrm{II}}}{\tau^{0} \sqrt{a}}$ & $\frac{K_{\mathrm{II}}^{0}}{\tau^{0} \sqrt{a}}$ & $\frac{K_{\mathrm{II}}}{\tau^{0} \sqrt{a}}$ & $\frac{K_{\mathrm{II}}^{0}}{\tau^{0} \sqrt{a}}$ & $\frac{K_{\mathrm{III}}}{\tau^{0} \sqrt{a}}$ & $\frac{K_{\mathrm{III}}^{0}}{\tau^{0} \sqrt{a}}$ & $\frac{K_{\mathrm{III}}}{\tau^{0} \sqrt{a}}$ & $\frac{K_{\mathrm{III}}^{0}}{\tau^{0} \sqrt{a}}$ \\
\hline 0 & 0.0000 & 0.0000 & 0.0000 & 0.0000 & -0.3161 & -0.5144 & -0.31644 & -0.51447 & 0.0000 & 0.0000 & 0.00000 & 0.00000 \\
\hline 30 & 0.0000 & 0.0000 & 0.0000 & 0.0000 & -0.3738 & -0.4455 & -0.37391 & -0.44554 & 0.1079 & 0.1929 & 0.10808 & 0.19292 \\
\hline 60 & 0.0000 & 0.0000 & 0.0000 & 0.0000 & -0.2786 & -0.2572 & -0.27845 & -0.25723 & 0.3186 & 0.3341 & 0.31885 & 0.33416 \\
\hline 90 & 0.0000 & 0.0000 & 0.0000 & 0.0000 & 0.0000 & 0.0000 & 0.00000 & 0.00000 & 0.3907 & 0.3858 & 0.39088 & 0.38585 \\
\hline 120 & 0.0000 & 0.0000 & 0.0000 & 0.0000 & 0.2786 & 0.2572 & 0.27845 & 0.25723 & 0.3186 & 0.3341 & 0.31885 & 0.33416 \\
\hline 150 & 0.0000 & 0.0000 & 0.0000 & 0.0000 & 0.3738 & 0.4455 & 0.37391 & 0.44554 & 0.1079 & 0.1929 & 0.10808 & 0.19292 \\
\hline 180 & 0.0000 & 0.0000 & 0.0000 & 0.0000 & 0.3161 & 0.5144 & 0.31644 & 0.51447 & 0.0000 & 0.0000 & 0.00000 & 0.00000 \\
\hline
\end{tabular}




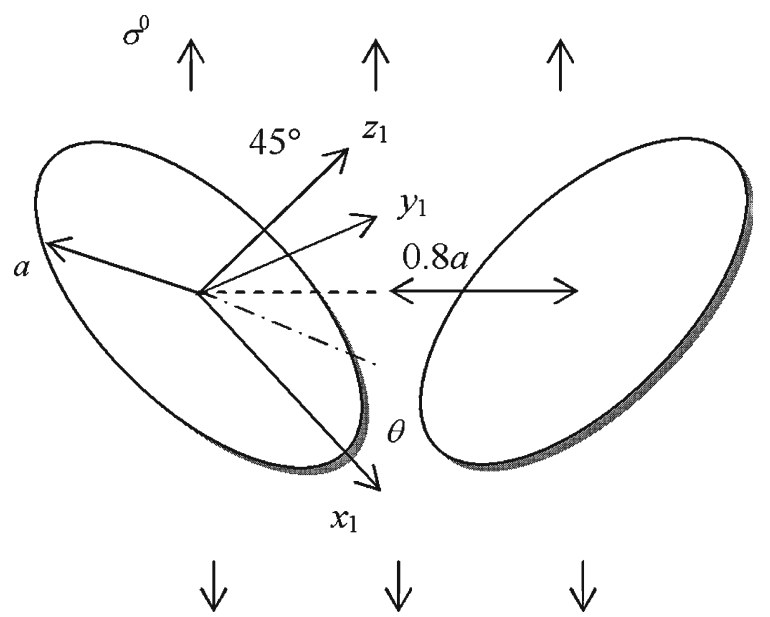

Fig. 6 "V" arrangement cracks under normal loading

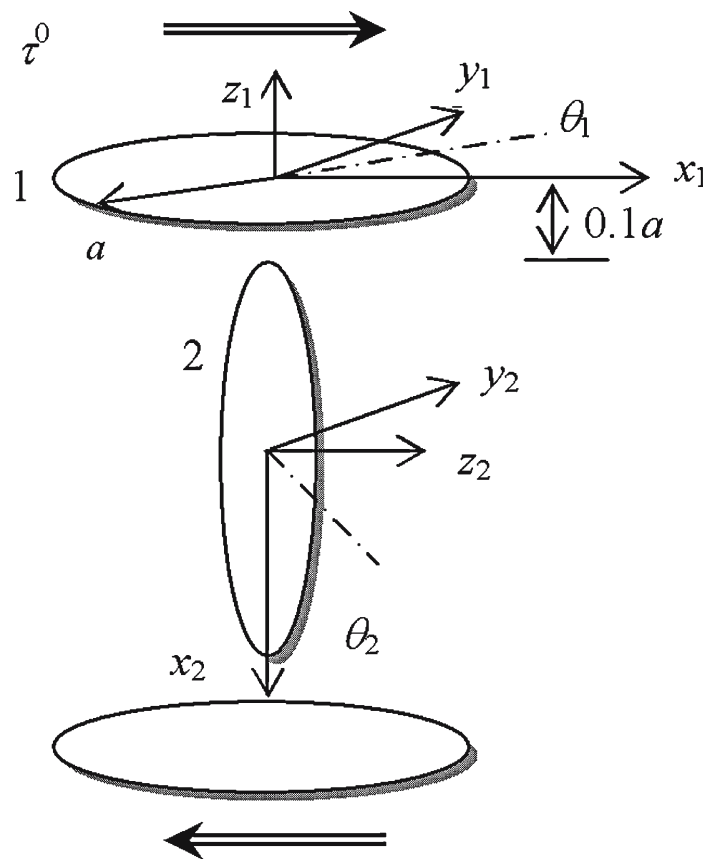

Fig. 7 "H" arrangement cracks under shear loading

for non-interacting cracks. We can see that the agreement is quite good. For "V" crack configuration, interaction produces a noticeable mode I SIF and a relatively weak effect on $K_{\text {II }}$ and $K_{\text {III }}$. For "H" crack configuration, the interaction results in a substantial amplification of $K_{\mathrm{I}}$ for the side cracks and the median crack is mildly shielded by the side cracks. The maximal value of $K_{\mathrm{II}}$ occurs at the points of the crack edge that are not the points of the smallest spacing.

\section{Conclusions}

Using oblate spheroidal coordinates and series expansions of displacement functions, an analytic method is developed to solve the problem of strong interacting and arbitrarily distributed penny-shaped cracks in threedimensional solids. The SIFs are calculated for several crack problems by using the boundary collocation procedure and the average method for crack-surface traction. When the first order average traction method is employed, our results are in good agreement with those of Kachanov [20] for several crack configurations which indicates that the present method is accurate and efficient for evaluating the SIFs. In addition, more order averages can be used in the present method. Numerical results show that the accuracy of the first order average traction method is high for large spacing; more order averages must be used for very closely spaced cracks.

\section{Appendix Oblate spheroidal coordinates (Gladwell [23])}

The oblate spheroidal coordinates $\xi, \eta, \theta$ are related to Cartesian coordinates $x, y, z$ by the equations

$$
\begin{aligned}
& x=a \sqrt{\left(1+\xi^{2}\right)\left(1-\eta^{2}\right)} \cos \theta, \\
& y=a \sqrt{\left(1+\xi^{2}\right)\left(1-\eta^{2}\right)} \sin \theta, \\
& z=a \xi \eta,
\end{aligned}
$$

where $-1 \leq \eta \leq 1, \xi \geq 0$. The surface $\xi=$ const, $\eta=$ const are the ellipsoids and hyperboloids (of one sheet)

$$
\begin{aligned}
& \frac{r^{2}}{a^{2}\left(1+\xi^{2}\right)}+\frac{z^{2}}{a^{2} \xi^{2}}=1, \\
& \frac{r^{2}}{a^{2}\left(1-\eta^{2}\right)}-\frac{z^{2}}{a^{2} \eta^{2}}=1,
\end{aligned}
$$

respectively; the surfaces $\xi=0$ and $\eta=0$ are the interior and exterior of the circle $r=a, z=0$, respectively.

Laplace's equation in oblate spheroidal coordinates is

$$
\begin{gathered}
\frac{\partial}{\partial \xi}\left[\left(1+\xi^{2}\right) \frac{\partial \psi}{\partial \xi}\right]+\frac{\partial}{\partial \eta}\left[\left(1-\eta^{2}\right) \frac{\partial \psi}{\partial \eta}\right] \\
+\frac{\left(\xi^{2}+\eta^{2}\right)}{\left(1+\xi^{2}\right)\left(1-\eta^{2}\right)} \frac{\partial^{2} \psi}{\partial \theta^{2}}=0,
\end{gathered}
$$

which has separable solutions

$$
\psi(\xi, \eta, \theta)=\Xi(\xi) H(\eta) \Theta(\theta),
$$


where $\Xi, H, \Theta$ satisfy

$$
\begin{aligned}
& \frac{\mathrm{d}}{\mathrm{d} \xi}\left[\left(1+\xi^{2}\right) \frac{\mathrm{d} \Xi}{\mathrm{d} \xi}\right]-\left[n(n+1)-\frac{m^{2}}{1+\xi^{2}}\right] \Xi=0, \\
& \frac{\mathrm{d}}{\mathrm{d} \eta}\left[\left(1-\eta^{2}\right) \frac{\mathrm{d} H}{\mathrm{~d} \eta}\right]+\left[n(n+1)-\frac{m^{2}}{1-\eta^{2}}\right] H=0, \\
& \frac{\mathrm{d}^{2} \Theta}{\mathrm{d} \theta^{2}}=-m^{2} \Theta
\end{aligned}
$$

The required periodicity of $\Theta(\theta)$ and the convergence of the solutions $\Xi, H$ require that $m, n$ be integers so that

$$
\psi(\xi, \eta, \theta)=\left\{\begin{array}{c}
P_{n}^{m}(i \xi) \\
Q_{n}^{m}(i \xi)
\end{array}\right\} \cdot\left\{\begin{array}{c}
P_{n}^{m}(\eta) \\
Q_{n}^{m}(\eta)
\end{array}\right\} \cdot\left\{\begin{array}{c}
\cos m \theta \\
\sin m \theta
\end{array}\right\}
$$

where $P_{n}^{m}, Q_{n}^{m}$ are the Legendre functions of the first and second kinds, respectively, and $m \leq n$.

Now consider which of the combinations shown in Eq. (44) is valid to represent $\psi . P_{n}^{m}(\mathrm{i} \xi)$ is unbounded at $\xi=\infty(r=\infty, z=0)$, while $Q_{n}^{m}(\eta)$ has logarithmic singularities at $\eta= \pm 1(r=0, z=0)$; both cases should, therefore, be excluded. This leaves the combination

$\psi(\xi, \eta, \theta)=Q_{n}^{m}(\mathrm{i} \xi) \cdot P_{n}^{m}(\eta)\left(b_{m n} \cos m \theta+c_{m n} \sin m \theta\right)$,

where $b_{m n}, c_{m n}$ are coefficients.

\section{References}

1. Kachanov, M.: Effective elastic properties of cracked solids: critical review of some basic concepts. Appl. Mech. Rev. 45, 304-335 (1992)

2. Kachanov, M.: Elastic solids with many cracks and related problems. In: Hutchinson, J., Wu, T. (eds.) Advances in applied mechanics. vol. 30, pp. 256-426 Academic, New york (1994)

3. Nemat-Nasser, S., Hori, M.: Micromechanics: Overall Properties of Heterogeneous Materials. North-Holland, Amsterdam (1993)

4. Krajcinovic, D.: Damage Mechanics. Elsevier, Amsterdam (1996)

5. Budiansky, B., O'Connell, R.J.: Elastic moduli of a cracked solid. Int. J. Solids Struct. 12, 81-97 (1976), DOI:10.1016/00207683(76)90044-5

6. Christensen, R.M., Lo, K.H.: Solutions for effective shear properties in three phase sphere and cylinder models. J. Mech. Phys. Solids 27, 315-330 (1979)

7. Hashin, Z.: The differential scheme and its application to cracked materials. J. Mech. Phys. Solids 36, 719-734 (1988)
8. Mori, T., Tanaka, K.: Average stress in matrix and average elastic energy of materials with misfitting inclusions. Acta Metall 21, 571-574 (1973)

9. Collins, W.D.: Some coplanar punch and crack problems in three-dimensional elastostatics. Proc. R. Soc., Series A 274, 507-528 (1963)

10. Andreikiv, A.E., Panasyuk, V.V.: Elastic equilibrium of a body weakened by a set of circular cracks situated along a plane. Soviet Physics Doklady, 16, 255-256 (1971)

11. O'Donoghue, P.E., Nioshika, T., Atluri, S.N.: Multiple coplanar embedded elliptical cracks in an infinite solid subject to arbitrary crack face tractions. Int. J. Num. Meth. Eng. 21, 437-449 (1985), DOI:10.1002/nme.1620210305

12. Isida, M., Hirota, K., Noguchi, H., Yoshida, T.: Two parallel eliptical cracks in an infinite solid subjected to tension. Int. J. Frac. 27, 31-48 (1985), DOI: 10.1007/BF00017211

13. Xiao, Z.M., Lim, M.K., Liew, K.M.: Stress intensity factors for two coplanar penny-shaped cracks under uniaxial tension. Int. J. Eng. Sci. 32, 303-311 (1994), DOI:10.1016/00207225(94)90010-8

14. Xiao, Z.M., Lim, M.K., Liew, K.M.: Stress intensity factors of two internal elliptical cracks in three-dimensional solid. Eng. Frac. Mech. 50, 431-441 (1995), DOI:10.1016/00137944(94)00244-c

15. Xiao, Z.M., Liew, K.M., Lim, M.K.: Interaction between a spherical inhomogeneity and two symmetrically placed penny-shaped cracks. Int. J. Frac. 70, 305-320 (1995), DOI: 10.1007/BF00032449

16. Xiao, Z.M., Lim, M.K., Liew, K.M.: Determination of stress field in an elastic solid weakened by parallel pennyshaped cracks. Acta. Mech. 114, 83-94 (1996), DOI: 10.1007/BF01170397

17. Xiao, Z.M., Lim, M.K., Liew, K.M.: The stress field and intensity factor due to a craze formed at the equator of a spherical inhomogeneity. Mech. Mater. 14, 301-312 (1993), DOI:10.1016/0167-6636(93)90085-6

18. Fabrikant, V.I.: Close interaction of coplanar circular cracks in an elastic medium. Acta. Mech. 67, 39-59 (1987), DOI: 10.1007/BF01182121

19. Kachanov, M.: Elastic solids with many cracks: a simple method of analysis. Int. J. Solids Struct. 23, 23-43 (1987), DOI:10.1016/0020-7683(87)900030-8

20. Kachanov, M., Laures J.P.: Three-dimensional problems of strongly interacting arbitrarily located penny-shaped cracks. Int. J. Frac. 41, 289-313 (1989), DOI:10.1007/BF00018861

21. Sokolnikoff, I.S.: Mathematical Theory of Elasticity, McGrawHill New York (1956)

22. Kassir, M.K., Sih, G.C.: Three-dimensional crack problems, Mechanics of Fracture, vol. 2. Noordhoff, Leyden (1975)

23. Gladwell, G.M.L.: Contact problems in the classical theory of elasticity, SIJTHOFF \& NOORDHOFF, Leyden (1980)

24. Fabrikant, V.I.: Complete solution to some mixed boundary value problems in elasticity. In: Hutchinson, J., Wu, T. (eds.) Advances in Applied Mechanics, vol. 27, pp. 153-223 Academic, New York 